\title{
EL IMPACTO DE LA EMISIÓN ELECTRÓNICA DE LOS RECIBOS POR HONORARIOS EN LOS PROFESIONALES DOCENTES DE LA FACULTAD DE CONTABILIDAD
}

THE IMPACT OF ELECTRONIC ISSUANCE OF RECEIPTS FOR FEES IN THE SCHOOL TEACHERS' PROFESSIONAL ACCOUNTING

\section{Raúl Jesús Vergara Moncada *}

Docente Asociado de la Facultad de Ciencias - UNMSM

[Recepción: Julio de 2012/ Conformidad: Agosto de 2012]

\section{RESUMEN}

Actualmente casi todas las Administraciones tributarias del mundo, bajo una estructura del Centro Interamericano de Administraciones Tributarias (CIAT), están en un amplio desarrollo de la era Electrónica: primero con el uso voluntario de las Planillas Electrónicas, luego con el Programa de Libro Electrónico (PLE), nuevos formatos de libros y registros vinculados a asuntos tributarios, facturas electrónicas y, recientemente, la emisión electrónica de Recibos Por Honorarios. En el caso peruano no es ajeno que la Superintendencia Nacional de Administración Tributaria(SUNAT), ha implantado por el momento de manera voluntaria este sistema, por lo cual el objetivo del presente trabajo de investigación es determinar en qué medida esta nuevo sistema de digitalización impacta en los profesionales que laboran en la Facultad de Ciencias Contables de la UNMSM.

Esperamos contribuir a que más docentes utilicen este sistema simplificando el sistema administrativo y tomen conciencia de que se ahorraría en la impresión de comprobantes de pago. Consecuentemente se estaría contribuyendo con la preservación de nuestro medio ambiente ya que se evitaría que disminuya la producción de papeles y de esta manera contrarrestaríamos la indiscriminada tala de árboles en nuestro país.

Palabras Claves: Emisión Electrónica de Recibos Por Honorarios, SUNAT, Libros de Ingresos y Egresos Electrónicos.

\begin{abstract}
Currently, in almost all of the Tax Administrations under the structure of American Center of Tax Administration (CIAT) are in an extensive development of the first electronic age with the voluntary use of spreadsheets, then with the Electronic Program Book (PLE), new formats of books and records related to tax matters, and recently with electronic invoices. The Peruvian case is no stranger to the National Superintendence of Tax Administration (SUNAT), which has implemented for the time being in a volunteer manner. Thus, the aim of this investigative work is to determine to what extent this new scanning system impacts the professionals who work in the School of Accounting of San Marcos. Hoping more teachers use this system and simplifying the administrative system would contribute to preserving our environment since this would prevent an increase on paper production and thus counterbalance the indiscriminate cutting of trees in this country.
\end{abstract}

Keywords: Electronic Issue receipts for fees, SUNAT, Books Electronic Revenue and Expenditure

* Contador Público Colegiado Certificado. Maestrante en Contabilidad con mención en Política y Administración Tributaria. Email: rvergara64@yahoo.es. 


\section{INTRODUCCIÓN}

Actualmente las instituciones públicas de la mayoría de los Gobiernos Electrónicos (EGob) en el mundo en lo que concierne a la utilización de recursos informáticos por medio de la Internet se ha masificado. En nuestros actuales tiempos es indispensable el uso de este recurso para agilizar la gestión de las administraciones tributarias en el mundo. En nuestro caso en particular la Superintendencia Nacional de Administración Tributaria (SUNAT) ha emitido un dispositivo legal que brinda a los usuarios el Sistema de Emisión Electrónica, es decir la emisión de recibos electrónicos vía Internet para el cobro de sus honorarios; consecuentemente la SUNAT, aprovechando el avance de las tecnologías de la información y comunicación, busca generar ahorros en tiempo y dinero a favor del contribuyente facilitando el cumplimiento de las obligaciones tributarias y mejorando la competitividad de las empresas.

Todo esto lo lograremos al eliminar gastos como el de legalización, impresión, almacenamiento y seguridad que implicael registro de libros contables y tributarios de manera física, y adicionalmente también se logra ayudar a la protección del medio ambiente al no usar papel.

\section{Objetivo general:}

El objetivo general del Trabajo de Investigación es evaluar dicho impacto economico en los usuarios de este sistema.

\section{Objetivos específicos:}

-Evaluar el uso del Sistema de Emisión Electrónica de los Recibos por Honorarios de la SUNAT en los profesionales que laboran en la Facultad de Ciencias Contables de la
UNMSM.

-Evaluar el grado de adecuación del uso de la tecnología por parte de los docentes de la Facultad de Ciencias Contables en sus trabajo independiente para efecto de cobro por sus honorarios

-Evaluar el impacto económico en los docentes de la Facultad de Ciencias Contables de la UNMSM que utilizan el sistema de Emisión Electrónica de Recibos por Honorarios profesionales y el Libro de Ingresos y Gastos Electrónicos implementado por la SUNAT.

\section{DISEÑO EXPERIMENTAL}

En nuestro trabajo de investigación se utilizara el diseño experimental en la Universidad Nacional Mayor de San Marcos.

El propósito del método experimental es investigar las posibles reacciones causa-efecto, exponiendo a uno o más grupos experimentales la acción de dos o más condiciones de tratamiento, comparando los resultados con uno o más grupos de control que no reciben el tratamiento.

\section{Metodología de investigación del proyec- to:}

\section{Descriptiva}

Son los que buscan especificar las propiedades importantes de personas, grupos, empresas, comunidades, o cualquier otro fenómeno que sea sometido a análisis.

La descripción lleva al investigador a presentar los hechos tal como ocurren.

El método descriptivo tiene como objetivo describir y analizar sistemáticamente "lo que existe" con respecto a las variaciones o a las 
condiciones de una situación. En estos estudios se obtiene información acerca de las características y el comportamiento actual dentro de un periodo corto de tiempo de los fenómenos, hechos o sujetos.

En nuestro presente trabajo de investigación se describirá la problemática existente respecto a la evaluación del impacto económico de la Emisión Electrónica de los Recibos por Honorarios en los profesionales docentes de la Facultad de Contabilidad.

\section{Explicativa}

Se utilizará el método explicativo, se acopiará información, se tamizará la información. La técnica ha utilizarse será con elaboración de Cuestionarios. El tamaño de la muestra la constituirá los docentes de la Facultad de Ciencias Contables de la UNMSM.

\section{MARCO TEÓRICO}

\section{Recibo por Honorarios Electrónicos}

El Recibo por Honorarios que emite por medio de SUNAT Operaciones en Línea SOL el trabajador independiente que preste sus servicios por el ejercicio individual de su profesión, arte, ciencia u oficio. Estos ingresos generan rentas de cuarta categoría, de acuerdo a la legislación del Impuesto a la Renta.

El Recibo por Honorarios Electrónico (RHE) no es un nuevo comprobante de pago, es el mismo Recibo por Honorarios que se emite de manera física, con la diferencia que en este caso su generación será a través del Sistema de Emisión Electrónica (SEE), teniendo como características:

Rentas de cuarta categoría: las generadas por el trabajo independiente.
Otrso ingresos calificados como rentas de cuarta categoría

También se consideran en este tipo de rentas las retribuciones que perciben los directores de empresas, síndicos o liquidadores, mandatarios, gestores de negocios, albaceas, y las dietas de los regidores de municipalidades y consejeros de gobiernos regionales.

Asimismo, los ingresos que se perciban a través de los Contratos Administrativos de Servicios (CAS) en el sector público se consideran en esta categoría, conforme a lo establecido en el reglamento del Decreto Legislativo 1057.

\section{Ingresos no considerados como rentas de cuarta categoría}

Los que se obtienen por trabajar con un contrato de servicios normado por la legislación civil, en el cual el contratante designa el lugar $y$ el horario para que el contratado realice sus labores y además le proporciona los elementos de trabajo, asumiendo los gastos que demande el servicio.

\section{CONCEPTOS}

Documento Electrónico: Unidad básica estructurada de información registrada, publicada o no, utilizando sistemas informáticos.

Hash: Secuencia de bits de longitud fija obtenida como resultado de procesar un documento electrónico con un algoritmo, de tal manera que: el documento electrónico produzca siempre el mismo código de verificación cada vez que se le aplique dicho algoritmo.

\section{DE LOS COMPROBANTES DE PAGO}

\section{Comprobantes de Pago}


Se considera comprobante de pago todo documento que acredite la transferencia de bienes, entrega en uso o prestación de servicios, calificado como tal por la Superintendencia Nacional de Administración Tributaria (SUNAT). En ese sentido y de conformidad al artículo $2^{\circ}$ del Reglamento de Comproban- tes de Pago, aprobado mediante Resolución de Superintendencia No 007-99/SUNAT.

\section{Obligación de emitir comprobantes de} pago.

Se emiten estos de conformidad al artículo

\section{BASE LEGAL DE LOS COMPROBANTES DE PAGO ELECTRÓNICOS}

\begin{tabular}{|c|c|}
\hline $\begin{array}{l}\text { Aprueban normas para la emisión de boletos de } \\
\text { Transporte aéreo de pasajeros }\end{array}$ & $\begin{array}{l}\text { Resolución de Superintendencia N }{ }^{\circ} \\
\text { 166-2004/SUNAT }\end{array}$ \\
\hline $\begin{array}{l}\text { Regula nueva emisión de boletos } \\
\text { de viaje para transporte ferroviario de pasajeros }\end{array}$ & $\begin{array}{l}\text { Resolución de Superintendencia N }{ }^{\circ} \\
\text { 173-2008/SUNAT }\end{array}$ \\
\hline $\begin{array}{l}\text { Resolución de Superintendencia que implementa la } \\
\text { emisión electrónica del recibo por Honorarios y el } \\
\text { llevado del Libro de Ingresos y gastos de manera } \\
\text { electrónica }\end{array}$ & $\begin{array}{l}\text { Resolución de Superintendencia N }{ }^{\circ} \\
\text { 182-2008/SUNAT }\end{array}$ \\
\hline $\begin{array}{l}\text { Normas para la emisión por medios electrónicos de } \\
\text { comprobantes de pago por la prestación de servicios } \\
\text { aeroportuarios a favor de los pasajeros }\end{array}$ & $\begin{array}{l}\text { Resolución de Superintendencia N }{ }^{\circ} \\
\text { 182-2010/SUNAT }\end{array}$ \\
\hline $\begin{array}{l}\text { Resolución de superintendencia que amplía el Sistema } \\
\text { de Emisión Electrónica a la Factura y documentos } \\
\text { vinculados a ésta }\end{array}$ & $\begin{array}{l}\text { Resolución de Superintendencia N }{ }^{\circ} \\
\text { 188-2010/SUNAT }\end{array}$ \\
\hline $\begin{array}{l}\text { Posterga la entrada en vigencia de las disposiciones } \\
\text { que regulan el rechazo de la factura electrónica y la } \\
\text { emisión de notas de crédito y de débito electrónicas }\end{array}$ & $\begin{array}{l}\text { Resolución de Superintendencia N }{ }^{\circ} \\
\text { 052-2011/SUNAT }\end{array}$ \\
\hline $\begin{array}{l}\text { Modifican la Resolución de Superintendencia NN } 188 \text { - } \\
\text { 2010/SUNAT en cuanto a las condiciones para la } \\
\text { emisión de la Nota de Crédito y de Débito electrónicas }\end{array}$ & $\begin{array}{l}\text { Resolución de Superintendencia N }{ }^{\circ} \\
\text { 073-2011/SUNAT }\end{array}$ \\
\hline
\end{tabular}

10 del Decreto Ley No 25632 (Ley de Comprobantes de Pago) en los siguientes casos: En la transferencia de propiedad de bienes, como lo es en el caso de las ventas.

En la transferencia en uso, como ocurre en el caso de los arrendamientos.

En la prestación de servicios de cualquier naturaleza.

\section{Oportunidad de entrega de los compro- bantes de Pago.}

De conformidad al artículo 5o del Reglamen- to de Comprobantes de Pago se entregarán los comprobantes de pago:

a) En la transferencia de bienes muebles, en el momento en que se entregue el bien o en el momento en que se efectúe el pago, lo que ocurra primero.

b) En el caso de retiro de bienes muebles, en la fecha del retiro.

c) En la transferencia de bienes inmuebles, en la fecha en que se perciba el ingreso o en la fecha en que se celebre el contrato, lo que ocurra primero. 
d) En la transferencia de bienes, por los pagos parciales recibidos anticipadamente a la entrega del bien o puesta a disposición del mismo, en la fecha y por el monto percibido.

e) En la prestación de servicios, incluyendo el arrendamiento y arrendamiento financiero.

f) En el caso de los documentos emitidos por las empresas que desempeñan el rol adquirente en los sistemas de pago mediante tarjetas de crédito y/o débito emitidas por bancos e instituciones financieras o crediticias, domiciliados o no en el país, así como de los documentos emitidos por las empresas que desempeñan el rol adquiriente en los sistemas de pago mediante tarjetas de crédito.

\section{Excepciones a la emisión de compro- bantes de pago.}

De conformidad al artículo $7^{\circ}$ del Reglamento de Comprobantes de Pago se exceptúa de la obligación de emitir comprobante de pago por:

a) La transferencia de bienes o prestación de servicios a título gratuito efectuados por la Iglesia Católica y por las entidades pertenecientes al Sector Público Nacional (excepto empresas). b) La transferencia de bienes por causa de muerte y por anticipo de legítima.

c) La venta de diarios, revistas y publicaciones periódicas efectuadas por canillitas.

d) El servicio de lustrado de calzado y el servicio ambulatorio de lavado de vehículos.

e) Los ingresos que se perciban por las funciones de directores de empresas, albaceas, síndicos, gestores de negocios, mandatarios y regidores de municipalidades y actividades similares.

CAMBIOS EN LOS LIBROS ELECTRÓNICOS

Mediante la Resolución de Superintendencia 234-2006 se han incorporado cambios en el registro de los libros contables que implican mayor información a registrar. Estos cambios tienen un impacto directo en la cantidad de hojas a usar y, por tanto, en el costo de llevar los libros contables.

\section{¿QUE ES EL GOBIERNO ELECTRONI- CO EN LA SUNAT ?}

Para la SUNAT, el Gobierno Electrónico: es la continua optimización de sus procesos y servicios, transformando las relaciones internas y externas hacia su integración, con el uso racional y automatizado de la información". 
¿TIENE UNA IDEA DEL IMPACTO EN SU EMPRESA?

\begin{tabular}{l|c|c|}
\hline LIBROS & Sin la R.S. 234-2006 & Con la R.S. 234-2006 \\
\hline $\begin{array}{l}\text { Libro de Inventario y } \\
\text { Balances }\end{array}$ & 528 & 5,000 \\
\hline Libro Diario & 21,000 & 900,000 \\
\hline Libro Mayor & 21,000 & 780,000 \\
\hline Registro de Compras & 8,400 & 8,400 \\
\hline $\begin{array}{l}\text { Registro de Ventas e } \\
\text { Ingresos }\end{array}$ & 2,400 & 18,216 \\
\hline $\begin{array}{l}\text { Registro de Activos } \\
\text { Fijos }\end{array}$ & 5,000 & 10,000 \\
\hline Registro de Costos & 1,400 & 16,800 \\
\hline $\begin{array}{l}\text { Registro de Inventario } \\
\text { permanente valorizado }\end{array}$ & 59,782 & $1 \times 188,000$ \\
\hline Total Hojas & & 2926,416 \\
\hline
\end{tabular}

Elaboración: Propia / Fuente: Elaboración SUNAT

COSTO ASOCIADOS A LOS LIBROS

\begin{tabular}{|l|c|c|}
\hline COSTO & Sin la R.S. 234-2006 & Con la R.S. 234-2006 \\
\hline Hojas & 2,391 & 117,040 \\
\hline Legalización & 6,240 & 235,200 \\
\hline Tóner & 2,400 & 108,480 \\
\hline Almacenamiento & 374 & 14,112 \\
\hline Total SI. & 11,406 & 474,832 \\
\hline Total\$ & 4,074 & 169,583
\end{tabular}

Elaboración: Propia / Fuente: Elaboración SUNAT 


\section{¿QUE ES EL SISTEMA DE LIBROS ELECTRONICOS?}

Es un sistema desarrollado por la SUNAT para que los contribuyentes puedan llevar sus libros contables de forma electrónica.

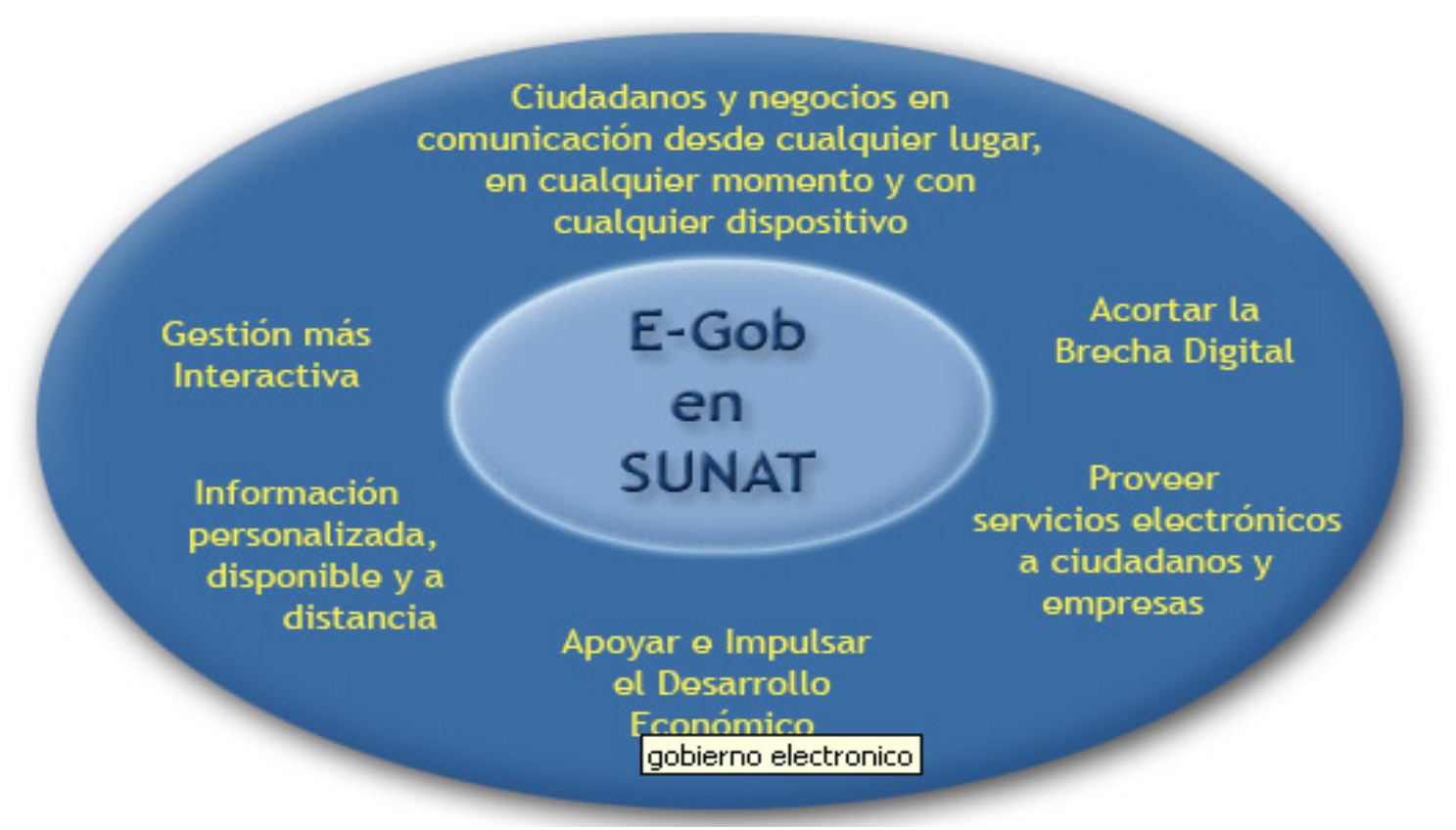

\section{¿CUÁLES SON LOS BENEFICIOS DE AFILIARSE AL SISTEMA DE LIBROS ELECTRÓNICOS?}

Eliminación de los costos de libros manuales u hojas sueltas o continuas: hojas, legalización del notario, impresión, almacenamiento físico y custodia.

Mayor eficiencia en los procesos de fiscalización: búsqueda, clasificación, e información contable y tributaria.

\section{MEJORA DE LA ECOLOGÍA EN NUES- TRO PAÍS.}

DATO: En el año 2009 se legalizaron $169^{`} 673,036$ hojas.

Con esa cantidad de hojas se podría haber cubierto la carretera de Tumbes a Tacna, ¡veinte veces!
Para fabricar esa cantidad de hojas:

Se cortaron 11,851 árboles de $25 \mathrm{~m}$. de altura y $20 \mathrm{~cm}$ de diámetro.

Se gastaron $84^{`} 649,878$ litros de agua limpia.

\section{¿Cómo emito Recibo por Honorarios Electrónicos?}

- Ingresar a emisión de recibo por honorarios electrónico.

- Emitir recibo por honorario electrónico.

¿Cómo genero los reportes de ingresos de cuarta categoría en el sistema de emisión electrónica?

De acuerdo a lo siguiente:

- Ingresar a reporte virtual de ingresos de cuarta categoría. 


\section{ESQUEMA DE AFILIACIÓN AL SISTEMA DE EMISIÓN ELECTRÓNICA}

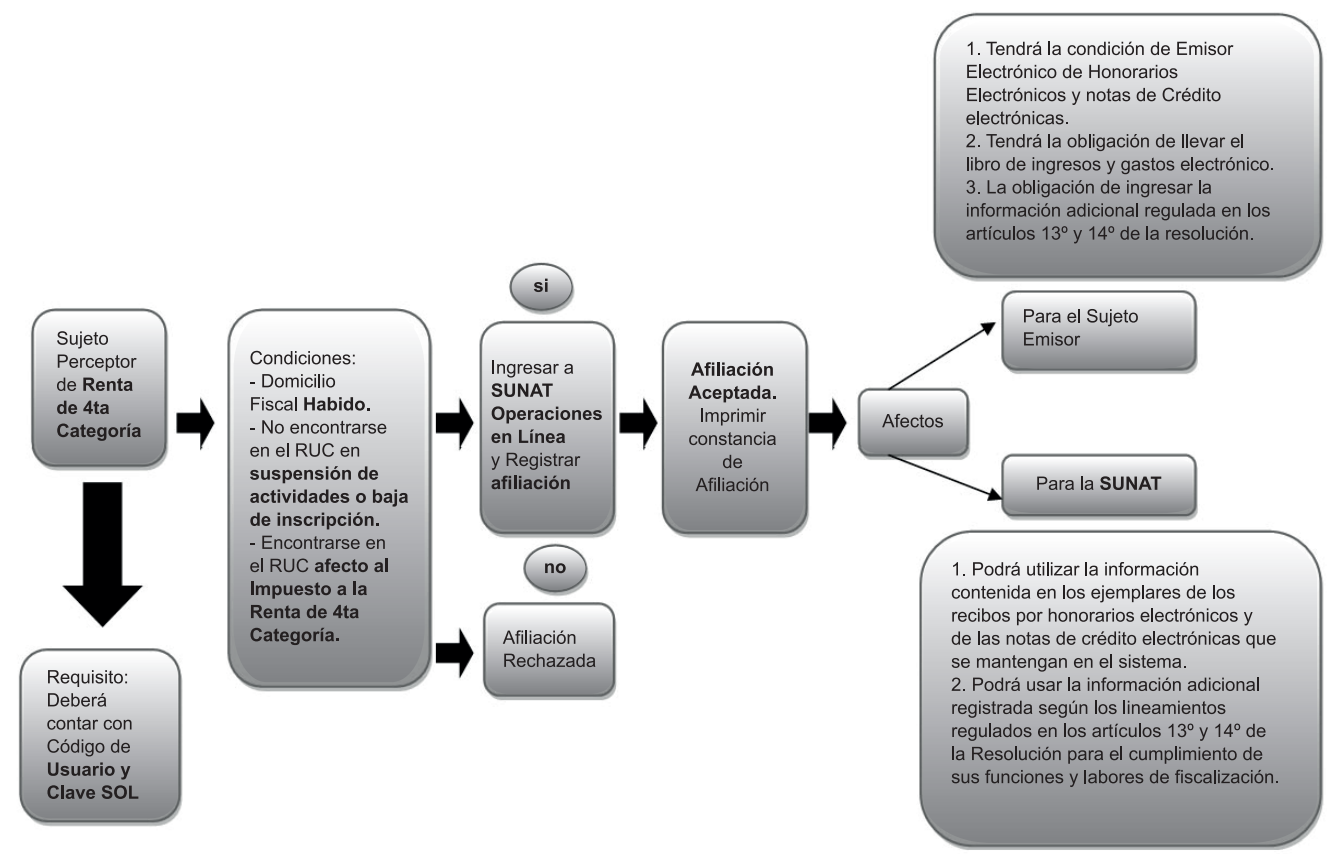

- Ingresar el periodo que desea consultar, indicando el mes y el año.

- Buscar

Todo profesional independiente tendría que emitir Recibos por Honorarios por internet

Según SUNAT a la fecha $(12 / 10 / 2011)$, 180,000 trabajadores independientes ya usan el sistema de Recibos por Honorarios Electrónicos.

Los profesionales que más lo utilizan son los abogados, ingenieros, contadores, y profesores; desde Enero del 2012 los trabajadores del Estado bajo el régimen del CAS también usan este sistema.

\section{PLANTEAMIENTO DE LAS PREGUN- TAS DE INVESTIGACION}

El problema principal

¿Cómo utilizan los docentes de la Facultad de Ciencias Contables de la UNMSM el Sistema de Emisión Electrónica de Recibos por Honorarios en su trabajo independiente? impacto económico del registro de ingreso y gastos electrónicos implementado por la SUNAT

Los Subproblemas

- ¿Cómo utilizan los docentes de la Facultad de Ciencias Contables de la UNMSM el Sistema de Emisión de Recibos por Honorarios Electrónicos en su trabajo independiente?

- ¿Cuál es el grado de adecuación del uso de la tecnología por parte de los docentes de la Facultad de Ciencias Contables en sus trabajo independientes para efecto de cobro por sus honorarios?

- ¿Cuál es el impacto económico en los docentes de la Facultad de Ciencias Contables de la UNMSM que utilizan el Sistema de Emisión Electrónica de Recibos por Honorarios profesionales y el Libro de Ingresos y Gastos Electrónicos implementado por la SUNAT? 


\section{PLANTEAMIENTO DE HIPOTESIS}

\section{Hipótesis general}

Los docentes de la Facultad de Ciencias Contables de la UNMSM utilizan adecuadamente el Sistema de Emisión de Recibos por Honorarios Electrónicos en su trabajo independiente. Grado de adecuación, e impacto económico del libro de Ingreso e Gastos Electrónicos implementado por la SUNAT

\section{Hipótesis Específica}

- Los docentes de la Facultad de Ciencias Contables de la UNMSM utilizan adecuadamente el Sistema de Emisión de Recibos por Honorarios Electrónicos en su trabajo independiente.

- Los docentes de la Facultad de Ciencias Contables de la UNMSM tienen un buen grado de adecuación del Sistema de Emisión Electrónica de Recibos por Honorarios y el Libro de Ingresos y Gastos Electrónicos-SUNAT.

- Los docentes de la Facultad de Ciencias Contables de la UNMSM perciben significativamente el impacto económico de la adecuación del Sistema de Emisión Electrónica de Recibos por Honorarios y el Libro de Ingresos y Gastos Electrónicos de la SUNAT

POBLACIÓN EN ESTUDIO Y SELECCIÓN DE LA MUESTRA

\section{DETERMINACIÓN DE LA POBLA- CIÓN EN ESTUDIO}

Se ha tenido en cuenta a los docentes de la Facultad de Contabilidad UNMSM.

\section{DETERMINACION DEL MARCO MUESTRAL}

En el presente trabajo de investigación se ha identificado, seleccionado y determinado el marco muestral de los docentes de la Facultad de la UNMSM

\section{MÉTODOS Y RESULTADOS (Encuesta y resultados de las mismas). \\ TECNICAS DE RECOLECCIÓN, DE MEDICIÓN Y DE ANÁLISIS DE LOS DATOS}

Elaborar los instrumentos de recolección de datos.

De las fuentes de información primarias y Secundarias, los datos que se obtengan mediante lectura, observaciones, conversaciones, preguntas, y grabaciones se recogerán utilizando los siguientes instrumentos y procedimientos de recolección de la información.

a) Fichas bibliográficas

Se registrarán la información y estadística obtenidas de las fuentes primarias que corresponden a la literatura revisada, instalaciones del sistema inalámbrico en las diferentes Facultades

b) Entrevistas

Los datos se registrarán en cuestionarios registrados, estructurados y preparados por el investigador, respondido por el entrevistado y llenado por el investigador.

Los entrevistados serán los docentes de la Facultad de Contabilidad de la UNMSM.

c) Encuestas

La información se registrará en Formularios preparados por el investigador y 
llenados por el encuestado que serán los docentes de la Facultad de Ciencias Contables de la UNMSM.

d) Entrevistas Cerradas

Grabadas en medios magnéticos, previa consentimiento del entrevistado, que será aplicado a los docentes, de la Facultad de la UNMSM.

Probar la validez y confiabilidad de los instrumentos.

a) Consulta de datos estadísticos Se realizarán consultas a las fuentes de Información de la SUNAT respecto a los docentes de la Facultad de Ciencias Contables de la UNMSM

Determinar la validez y la confiabilidad de las mediciones de los datos. Se tomará en cuenta los datos tabulados.

Crear la base de archivos de datos. Se creará una Base de Datos totalmente codificados en una hoja electrónica.

\section{ANÁLISIS DE LOS DATOS}

SELECCIONAR LAS MEDICIONES Y PRUEBAS ESTADÍSTICAS

Para las variables cuantitativas las principales medidas de tendencia central y dispersión.

ELABORAR EL PROGRAMA DE ANÁLISIS DE VARIABLES

Los datos de las variables se recopilarán mediante la coordinación con los miembros integrantes del equipo de trabajo.
ANEXOS (ENCUESTAS)

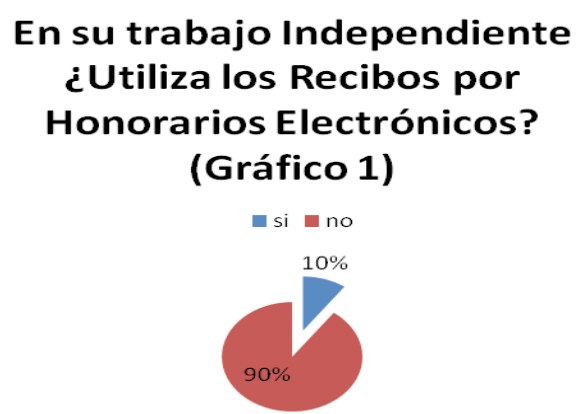

¿Es fácil para Ud.? adecuarse a los nuevos Sistemas de Emisión Electrónica de la SUNAT (Gráfico 2)

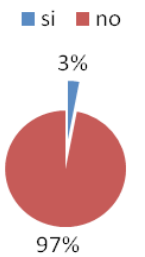

¿Percibe Ud. significativamente? el Impacto Economico del Sistema de Emision Electronica -SUNAT Gráfico 3)

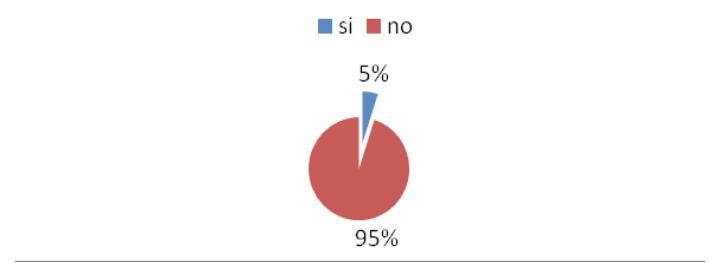

Si Ud. sabe que existen los Recibos por Honorarios Electronicos ¿Porque no se inscribe en el Sistema de Emisión Electrónica - SEE (Gráfico 4)

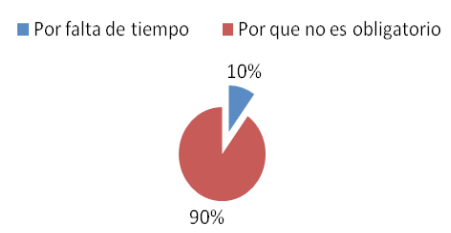




\section{CONCLUSIONES}

- Un $90 \%$ de los docentes encuestados de la Facultad de Ciencias Contables no utiliza los Recibos por Honorarios Electrónicos por que se dedica exclusivamente a la docencia en calidad de perceptores de renta de quinta categoría, y solo un $10 \%$ que realiza actividades adicionales de cuarta categoría como trabajadores independientes utiliza este nuevo Sistema de Emision Electronica - SUNAT.

- Del 10\% de Docentes de la Facultad de Ciencias Contables que emiten su Recibo por Honorarios Electronicos solo un 03 por ciento les ha sido fácil adecuarse a los nuevos Sistemas de Emision Electronica - SUNAT, por que han tenido que ser asesorados por funcionarios de la administracion pública para afiliarse a este nuevo sistema.

- De los docentes de la Facultad de Ciencias Contables que emiten Recibos por Honorarios Electrónicos como trabajadores Independientes un $95 \%$ perciben que la minimizacion de sus costos no son muy significativos porque el volumen de sus ingresos por asesorías es mínimo ya que en ciertos casos emiten 03 o 04 recibos mensuales, esto es variable.

- Un $90 \%$ de los docentes de la Facultad de Ciencias Contables de la UNMSM que realizan trabajos adicionales como independientes gira actualmente Recibos por Honorarios físicos, y no se inscriben al Sistema de Emision Electronica por que es optativo.

\section{RECOMENDACIONES}

- Que se dé mayores facilidades a los que emiten Recibos por Honorarios Electrónicos en los registros de ingresos y gastos electronicos en su calidad de perceptores de renta de cuarta categoría.

- Que los docentes de la Facultad de Ciencias Contables que generan adicionalmente renta de cuarta categoría sean capacitados en la facultad por funcionarios de la administracion tributaria para la correcta generacion de re- portes a través de este sistema y la utilizacion de las notas de crédito electrénicas en el libro de ingresos y gastos electrónicos.

- Que se difunda los beneficios económicos que conlleva este sistema, al ya no tener que comprar los libros de ingresos y gastos, ahorro en la legalización de los costos de la notaría, almacenamiento de informacion, rapides para localizar informacion detallada, evitarse pérdida de tiempo acudiendo presencialmente a la SUNAT.

- Sensibilizar a los docentes de la Facultad de Ciencias Contables de la UNMSM que adicionalmente son perceptores de renta de cuarta categoría por su trabajo independiente, en el tema de los registros electronicos.

\section{REFERENCIAS BIBLIOGRÁFICAS}

1. Gonzales Lopez, Oscar Rodrigo (2009), Internet para la Empresa. Ed. Anaya Multimedia.

2. Neg Zheng Elisa (2006) "Internet es una gigantesca red”. Extraído el 10 abril del 2010 de www.angelfire.com/ak5/interneto

3. Kozma, Robert B., (2008) "Tecnologías de la información y la comunicación, reforma educativa y crecimiento ecónomico", en revista $\mathrm{Az}$, No 10 pp.86-89

4. Apaza Meza, Mario (2010). Aplicación práctica del impuesto a la renta. Ejercicio 20102011, Pacifico Editores.

5. Caro Aturo, Salas Octavio, (2008) "Second Life Alfa y omega 113 pág.

6. Web site: http://www.sunat.gob.pe

7. Informativo Caballero y Bustamante (2008) 2da Quincena octubre 2008 pp. $\mathrm{B} 1, \mathrm{~B} 2, \mathrm{~B} 3, \mathrm{~B} 4$.

8. Actualidad empresarial (2008) No $1692 \mathrm{da}$ Quincena octubre 2008, pp. I-23,I-24,I25,I-26.

9. Diario Gestión, Miércoles 12 de Octubre 2011.pp. $1-2$. 